\title{
Is it always anterior chest pain angina pectoris?
}

\author{
Viviana Aursulesei ${ }^{* 1,2}$, Ana-Maria Buburuz ${ }^{1,2}$, Siminela Bulughiana ${ }^{2}$, Irina luliana \\ Costache ${ }^{1,2}$
}

1"Grigore T. Popa" University of Medicine and Pharmacy lași, Romania, ${ }^{2}$ Cardiology Department, "Sf. Spiridon" University County Emergency Hospital, Iaşi, Romania

\begin{abstract}
Acute pulmonary embolism (PE) still remains a difficult diagnosis because of its chameleonic clinical presentation. Although it is a common clinical scenario, PE is frequently underdiagnosed. We report a case of acute PE that highlights the importance of a diagnostic strategy based on clinical probability assessed by a validated prediction rule. Our report is also highly suggestive for the importance of computed tomographic angiography, the most useful imaging method that provide direct signs for diagnosis and also significant information on alternative clinical conditions. A useful conclusion for clinical practice is that any data must be rationally judged, considering the limits of clinical diagnosis and laboratory tests.
\end{abstract}

Keywords: pulmonary embolism, angina pectoris, computed tomographic angiography

\section{Introduction}

Acute pulmonary embolism (PE) still remains a challenge for clinician, especially when shock or hypotension is absent. Although breathing difficulty and chest pain are the most common symptoms, the diagnosis cannot rely only on clinical data.

For an appropriate diagnosis the clinical probability index it must be considered, after excluding alternative conditions by using multiple diagnostic methods.

If misinterpreted any helpful information may raise other questions or uncertainties.

Received: April 2016; Accepted after review: June 2016; Published: June 2016.

*Corresponding author: Grigore T. Popa" University of Medicine and Pharmacy lași, Romania, ${ }^{2}$ Cardiology Department, "Sf. Spiridon" University County Emergency Hospital, 16 University Street, 700115, Iași, Romania.

E-mail: aursuleseiv@yahoo.com

\section{Case report}

A 72 year-old man, former heavy smoker, with type IIB dyslipidemia, was admitted for progressive dyspnea and chest pain. A month ago, the patient was hospitalized for retrosternal pain, shortness of breath, but also left side chest pain accompanied by left pleural friction rub and systemic inflammatory response (ESR of $84 \mathrm{~mm}$, fibrinogen of 780 $\mathrm{mg} / \mathrm{dl}$, C-reactive protein of $27 \mathrm{mg} / \mathrm{dl}$, leukocytosis of $13.600 / \mathrm{mm}^{3}, 77$ percent neutrophils).

Based on ECG changes during admission (Figures 1a and 1b) and presence of cardiovascular risk factors (old age, cholesterol of $254 \mathrm{mg} / \mathrm{dl}$, LDL-cholesterol of $138 \mathrm{mg} / \mathrm{dll}$, HDL-cholesterol of $35 \mathrm{mg} / \mathrm{dl}$ ), the diagnosis of stable angina was established.

Considering the left pleural friction rub and parameters of inflammation, the diagnosis of pleuritis sicca was also affirmed. During hospitalization and after discharge the patient was treated with Bisoprolol $2.5 \mathrm{mg} /$ daily, Isosorbide mononitrate $40 \mathrm{mg} /$ daily, 
Clopidogrel 75 mg/daily, Rosuvastatin 20 $\mathrm{mg} /$ daily, Ibuprofen $400 \mathrm{mg} /$ daily, Omeprazole $40 \mathrm{mg} /$ daily.

On current admission the main complaint was worsening dyspnea and anterior chest pain with equivocal characters for ischemic etiology as it was continuous and increased by right arm abduction. The physical examination found disseminated bronchial rales, no pleural friction rub, normal hemodynamic parameters (heart rate 84 beats per minute, blood pressure of $110 / 70 \mathrm{mmHg}$ ), varicose veins of left lower limb. ECG revealed inversion of $T$ waves in leads V1-V3 (Figure 2), with variable voltage on successive recordings. No elevation of cardiac markers was identified
(CK-MB of $14 \mathrm{U} / \mathrm{L}$, troponin I of $0.004 \mathrm{ng} / \mathrm{mL}$ ). Arterial blood gas analysis detected no hypoxaemia.

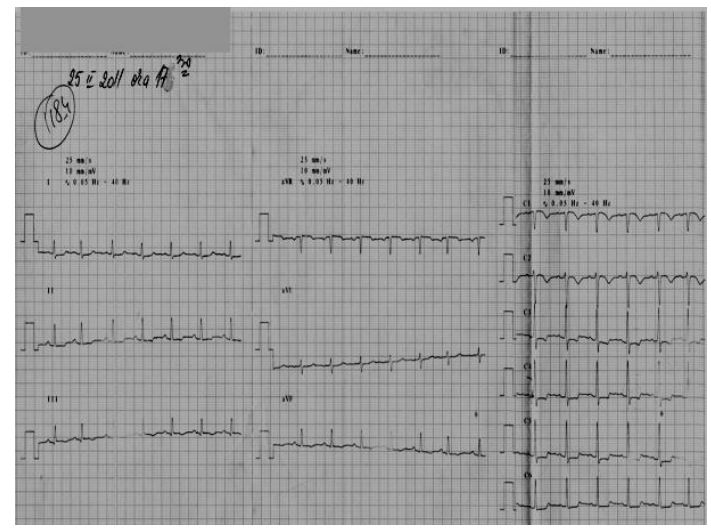

Fig. 1a. 12 leads ECG. Inversion of T waves in V1V2 and depression of ST segment followed by inverted $\mathrm{T}$ waves in V3/biphasic $\mathrm{T}$ waves $\mathrm{V} 4-\mathrm{V} 5$

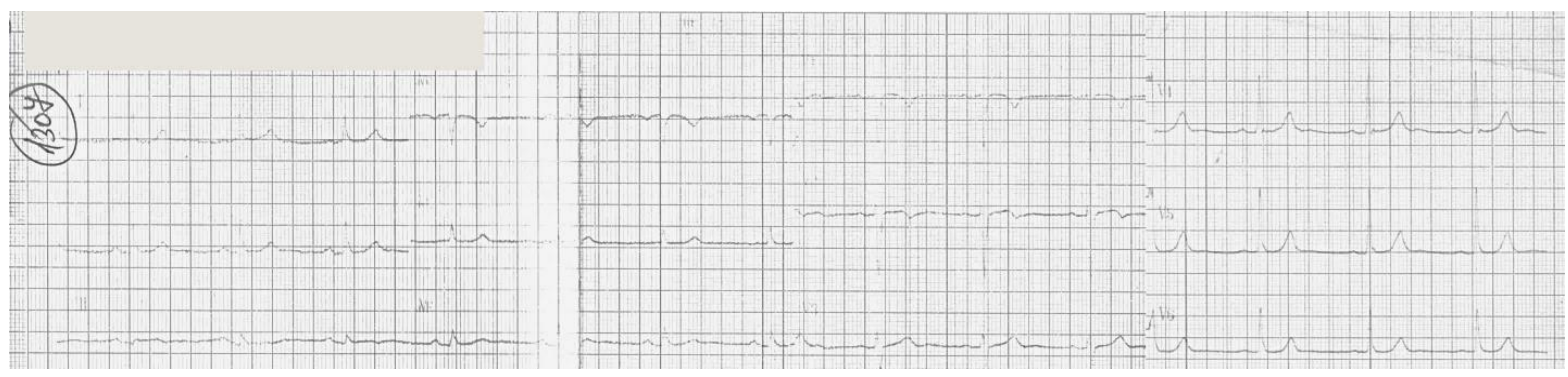

Fig. 1b. 12 leads ECG. Inversion of T waves in V1 and biphasic in V2

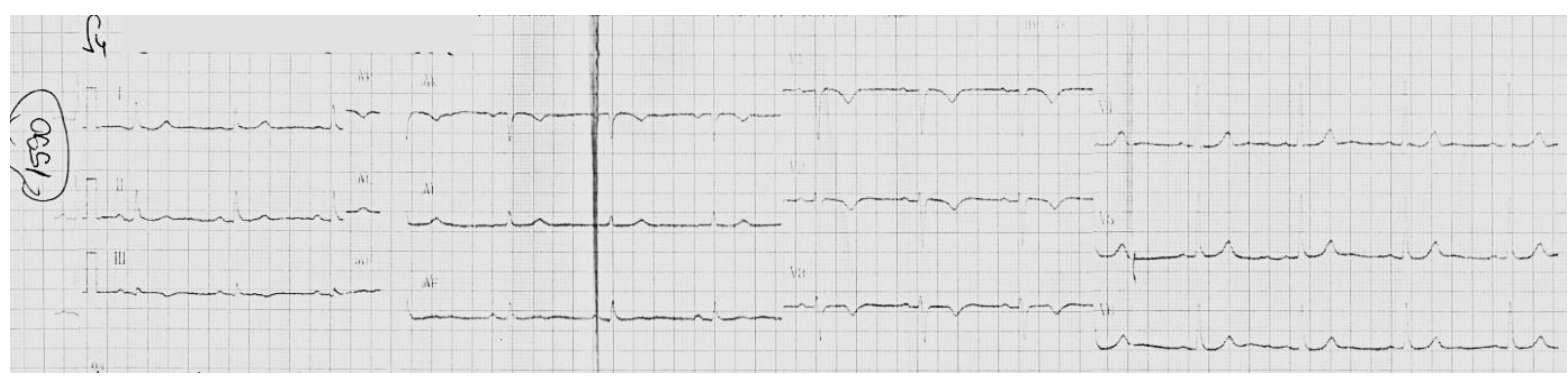

Fig. 2. 12 leads ECG. Inversion of $\mathrm{T}$ waves in V1-V3

The frontal chest X-ray showed a dilated right pulmonary artery and a normal-sized heart. The transthoracic echocardiography noted only mild dilatation of right ventricle (34 $\mathrm{mm}$ ) with no marker of ventricular dysfunction, while lower limb compression venous ultrasonography showed no sign of deep vein thrombosis (DVT). There were no arguments for an acquired thrombophilia (normal hemogram test and renal function, absence of lupus anticoagulant, anticardiolipin and anti- beta2-glycoprotein I antibodies). We had not the possibility to evaluate congenital thrombophilia.

Considering all above mentioned data the hypothesis of acute pulmonary embolism was raised. Based on the original version of revised Geneva and Wells scores for clinical prediction of PE [1], an intermediate clinical probability was suspected (clinical decision rule points 4 and 3 respectively) (Table 1). 
Table 1. Original version of Wells and revised Geneva scores (adapted from 2014 ESC Guidelines on the diagnosis and management of acute pulmonary embolism). The scores calculated for the patient suggested an intermediate clinical probability for acute PE.

\begin{tabular}{|c|c|c|c|}
\hline \multicolumn{2}{|l|}{ Revised Geneva score } & \multicolumn{2}{|l|}{ Wells score } \\
\hline Previous PE or DVT & 3 & Previous PE or DVT & 1.5 \\
\hline Heart rate & & Heart rate $\geq 100$ beats $/ \mathrm{min}$ & 1.5 \\
\hline 75-94beats/min & 3 & & \\
\hline$\geq 95$ beats/min & 5 & & \\
\hline $\begin{array}{l}\text { Surgery or fracture within the past } \\
\text { month }\end{array}$ & 2 & $\begin{array}{l}\text { Surgery/immobilization within the past } \\
\text { four weeks }\end{array}$ & 1.5 \\
\hline Haemoptysis & 2 & Haemoptysis & 1 \\
\hline Active cancer & 2 & Active cancer & 1 \\
\hline Unilateral lower limb pain & 3 & Clinical signs of DVT & 3 \\
\hline $\begin{array}{l}\text { Pain on lower limb deep venous } \\
\text { palpation and unilateral oedema }\end{array}$ & 4 & Alternative diagnosis less likely than PE & 3 \\
\hline Age $65>$ years & 1 & & \\
\hline Clinical probability - three-level score & & Clinical probability - three-level score & \\
\hline Low & $0-3$ & Low & $0-1$ \\
\hline Intermediate & $4-10$ & Intermediate & $2-6$ \\
\hline High & $\geq 11$ & High & $\geq 7$ \\
\hline
\end{tabular}

According to the proposed diagnostic algorithm for patients with suspected PE with no shock or hypotension and low/intermediate clinical probability [2], plasma D-dimer testing was performed and revealed elevated levels of $5852 \mathrm{ng} / \mathrm{mL}$. Multidetector computed tomographic (MDCT) angiography was the second-line test. Pulmonary artery thrombi in both principal arteries before bifurcation, with extension on lobar arteries and some of segmental arteries were documented (Figures $3 a$ and $3 b$ ). At the same time, the native thoracic CT described a ground-glass pattern of the superior lobe of right lung, consolidation of right apical segment and multiple indeterminate small nodules, together with mediastinal and some calcified infracentimetric hilar lymph nodes (Figure 3c).

Considering the pneumologist opinion, a fibrobronchoscopy was further needed for more precise characterization of CT pattern and also to exclude a lung cancer. In such a case, the cancer-related PE would be plausible.
The fibrobronchoscopic exam revealed a normal larynx and trachea, but the right upper lobe bronchial anatomy presented multiple blind fistulas, deviation of anterior segmental bronchus and narrowing of apical segmental bronchus with normal local mucosa. Negative local cultures for Koch's bacillus and nonspecific bacteria were obtained, but cytological exam showed malignant cells, necrosis and hemorrhage.

The opinion of a thoracic surgeon was requested in order to establish therapeutic attitude. The decision was to initiate anticoagulation therapy followed by a second thoracic CT one month later. The patient was treated with enoxaparin given subcutaneously at weight-adjusted doses $(80 \mathrm{mg} / \mathrm{kg}$ every 12 hours) for 7 days, with monitoring of the platelet count. Treatment with subcutaneous anticoagulant was followed by the administration of oral vitamin $\mathrm{K}$ antagonist (Acenocoumarol). The daily dose was adjusted according to the INR, targeting an INR level of 2.0-3.0. 


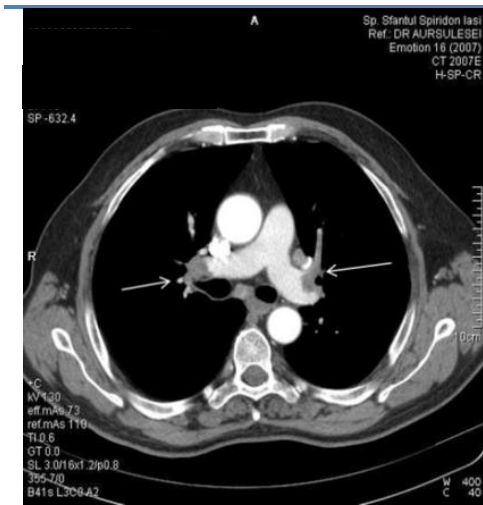

Fig. 3a. CT angiography. Pulmonary artery thrombi in both principal arteries before bifurcation (arrows).

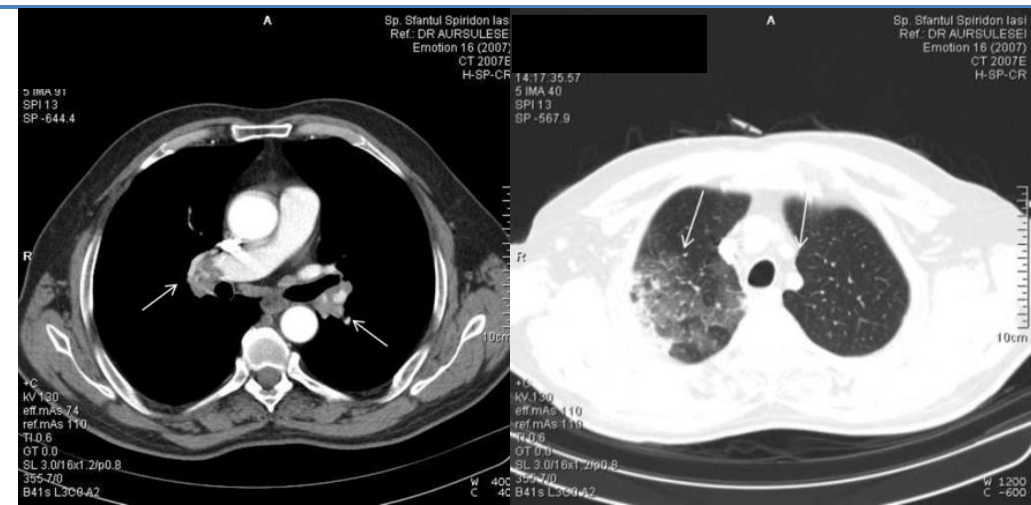

Fig. 3b. CT angiography. Fig. 3c. Native thoracic CT. Pulmonary artery thrombi in right Ground-glass attenuation of principal artery extending on superior lobe of right lung, lobar and segmental arteries consolidation of right apical (arrows). segment and multiple indeterminate small nodules, mediastinal and hilar infracentimetric lymph nodes (arrows).
Gastrointestinal protection was also assured by administration of Omeprazole 40 $\mathrm{mg}$ daily.

Clinical evolution was favorable at discharge. After a month, parameters of systemic inflammatory response regressed (ESR of $41 \mathrm{~mm}$, fibrinogen of $317 \mathrm{mg} / \mathrm{dl}$, Creactive protein of $1.34 \mathrm{mg} / \mathrm{dl}$, white blood cells of $6.750 / \mathrm{mm}^{3}$ ), CT angiography showed the same distribution of arterial thrombi, but a normal pattern of lung parenchyma was described by native CT.

Because we couldn't detect any strong or moderate predisposing factor according to ESC guidelines on acute PE [2], long-term oral anticoagulation was considered. Our patient still has an uncomplicated evolution after five years of oral anticoagulation, with no hemorrhagic event or PE recurrence. No cancer was diagnosed during this period.

\section{Discussions}

We reported a case of acute PE without shock or hypotension, a common clinical scenario, but frequently underdiagnosed. Also, we aimed to highlight the importance of recognizing this chameleonic condition that may impact the vital prognosis. From a clinical point of view it must be noted that clinical signs and symptoms are non-specific. Progressive or sudden dyspnea, pleuritic/anterior chest pain, tachypnea, cough or hemoptysis there are common clinical characteristics of patients with suspected PE [3].

Each of these signs or symptoms does not confirm but does not exclude PE. For this reason the most important step in determining the likelihood of PE is to use simple, standardized and validated prediction rules (Wells and Geneva). Clinical diagnosis can thus be optimized. Chest pain is a frequent symptom of $\mathrm{PE}$, but its clinical characters vary depending on the mechanism. In central PE chest pain may have a typical angina character reflecting a possible right ventricle ischemia [2].

Because our patient presented dynamic ECG anterior ischemic changes and cardiovascular risk factors, the confusion with angina pectoris occurred. Yet, when a patient has dyspnea without documented heart failure or pulmonary disease, PE should be considered. The clinical hypothesis was also sustained by the novel characters of chest pain during second admission. In this case, chest pain was suggestive for pleural irritation due to distal emboli causing pulmonary infarction. 
In one's turn, pulmonary infarction could be a rational explanation for both left pleural rub and left side chest pain described during first admission and also for the persistent systemic inflammatory response. In this new clinical context, inversion of $T$ waves in leads V1-V3 could be indicative of right ventricular strain [2]. ECG changes are non-specific for $\mathrm{PE}$, are usually found in more severe cases, but may complete the diagnosis when other clinical conditions are excluded. On the other hand, a normal ECG has no significant predictive value.

Chest X-ray is frequently non-specific in $P E$, but it is useful for excluding other causes of dyspnea and chest pain. A normal chest Xray in a patient with severe dyspnea, but without evidence of cardiomegaly or pleural, pulmonary changes, may be indicative of $\mathrm{PE}$.

According to ESC guidelines on acute PE, echocardiography is not recommended as a routine method in haemodynamically stable, normotensive patients with suspected PE (2). Conversely, in PE with shock or hypotension, providing unequivocal signs of right ventricle pressure overload and dysfunction, echocardiography justify emergency reperfusion treatment.

In blood gas analysis, hypoxemia may be a typical finding in acute PE, but up to $40 \%$ of the patients have normal arterial oxygen saturation [2].

It is important to highlight that the diagnosis of acute PE without shock or hypotension is still difficult in patients with no strong predisposing factors such as major trauma, lower limb fractures or surgery. According the classification of patient-related predisposing factors for PE [2], only some weak predisposing factors such as varicose veins and increasing age were detected in our patient. The new theory regarding the link between atherothrombosis and venous thromboembolism suggests the importance of common cardiovascular risk factors for a high risk of PE. Actually, our patient was a heavy smoker with untreated dyslipidemia. As ICOPER Registry observed [4] there are about 10-20 percent of the so-called unprovoked PE, when predisposing factors are not documented. In these cases, congenital or acquired thrombophilia and malignancy should be suspected. We didn't find any cause of acquired thrombophilia. Although necessary, the study of congenital thrombophilia was not available in our hospital at that time, and was also too expensive to be supported by our patient.

It is well documented that about 10 percent of patients have an occult cancer when PE is first diagnosed. Also, about 10 percent of patients presenting with unprovoked PE will develop cancer, mostly appearing in the first 1-2 years after diagnosis [5].

ESC guidelines on acute PE highlight that the etiology of $\mathrm{PE}$ is frequently multifactorial and may explain why a patient not presenting obvious provoking factors may be still at moderate/high risk of PE. A special discussion is required for the absence of deep vein thrombosis (DVT), considering that in $90 \%$ of patients $P E$ originates from vein clots in lower limbs. On the other hand, compression venous ultrasonography shows a DVT in only $30-50 \%$ of patients with PE, especially in those with distal clots. A possible explanation is the spontaneous resolution or embolization of small vein clots. On the other hand, the method of examination may be involved. A negative venous Doppler ultrasonography does not rule out DVT, because many DVTs occur in inaccessible areas for examination. As ESC guidelines on acute PE emphasize, in suspected PE ultrasonography scan can be limited to a simple four point examination (groin and popliteal fossa) [2].

The diagnostic yield may be increased further by performing complete ultrasonography, which includes the distal veins. Yet, in asymptomatic patients with distal vein clots, accuracy of method remains poor [6].

Multi-detector computed tomographic (MDCT) angiography has become the method of choice for imaging the pulmonary arteries in suspected PE. According to the validated strategy applied for patients with low/intermediate clinical probability and elevated D-dimer levels, MDCT angiography was the second-line test. The method was diagnostic because extensive arterial thrombi, including central arterial tree, were 
documented. The value of MDCT is augmented by its unique possibility to provide significant additional information related to alternative or associated clinical conditions. On the other hand, when sub-segmental defects are observed the diagnostic value and therapeutic attitude are still under debate [3].

At the same time, it is very important to properly interpret the imagistic information. In our case for example, semiological features of pulmonary infarction on CT were difficult to interpret, leading to a complicated diagnostic algorithm. Ground-glass attenuation can precede typical segmental consolidation and is suggestive for incomplete pulmonary infarction, but may be confused with inflammatory pulmonary lesions. Pseudotumoral or fibrous lesions can also be detected in this context. So, a pulmonary infarction may seriously change the bronchial anatomy as in the case of our patient. On the other hand, tumoral emboli may appear as a typical pulmonary infarction and can be hardly differentiate from another tumor. In pulmonary cancers of vascular tissue high-resolution CT also demonstrates irregular, ill-defined peribronchovascular nodules, with surrounding ground-glass attenuation. Clinical context and the patient tipology are highly suggestive in this situation [7]. All these data justify fibrobronchoscopy and cytological examination. Finally, our case demonstrates the difficulty of cytodiagnosis. There are many factors influencing the final result - an adequate sampling and preparation technique, the quality of the smear, but also the analyzing method and a good experience of pathologist.

It is also known that under different stimuli reactive mesothelial cells can proliferate rapidly and show certain malignancy criteria.

\section{References}

1. van Belle $A$, Büller HR, Huisman MV, et al. Effectiveness of Managing Suspected Pulmonary Embolism Using an Algorithm Combining Clinical Probability, D-Dimer Testing, and Computed Tomography. JAMA 2006; 295:172-179.

2. Konstantinides $S$, Torbicki $A$, Agnelli $G$, et al. 2014 ESC Guidelines on the diagnosis and
Inflammatory processes reinforce this effect so the diagnosis of malignancy should be established extremely carefully. Recognition of basic inflammatory cells and plasma cells is also essential for interpretation of cytology samples. Some tumors contain a large number of inflammatory cells, but these are very uncommon, even when tumor necrosis [8] is present. For this reason, a final positive diagnosis requires the correlation of clinical examination and laboratory investigations with cytological examination.

\section{Conclusions}

Although the diagnostic myth in PE is that the patient has breathing difficulty and chest pain, in reality the diagnosis cannot rely only on clinical data, although signs and symptoms should not be eluded. Laboratory tests don't replace clinical judgement. If appropriate methods are used, the diagnosis is completed in a safe manner for the patient. A prompt diagnosis is the best reward for physician but it is priceless for patient life.

\section{Consent}

Written informed consent was obtained from the patient for publication of this case report and accompanying images. A copy of the written consent is available for review by the Editor-in-Chief of this journal.

\section{Competing interests}

The authors declare that they have no competing interests.

management of acute pulmonary embolism. Eur Heart J 2014; 35:3033-3080.

3. Pollack CV, Schreiber D, Goldhaber SZ, et al. Clinical characteristics, management, and outcomes of patients diagnosed with acute pulmonary embolism in the emergency department: initial report of EMPEROR (Multicenter Emergency 
Medicine Pulmonary Embolism in the Real World Registry). J Am Coll Cardiol 2011; 57(6):700-706.

4. Goldhaber SZ, Visani L, De Rosa M. Acute pulmonary embolism: clinical outcomes in the International Cooperative Pulmonary Embolism Registry (ICOPER). Lancet 1999; 353(9162):13869.

5. Heit JA III, Silverstein MD, Mohr DN, et al. Risk factors for deep vein thrombosis and pulmonary embolism: a population based casecontrol study. Arch Intern Med 2000; 160(6):809815.

6. Ward MJ, Sodickson A, Diercks DB, Raja AS. Cost-effectiveness of lower extremity compression ultrasound in emergency department patients with a high risk of hemodynamically stable pulmonary embolism. Acad Emerg Med 2011; 18(1):22-31.

7. Hauret L, Minvielle F, Ehre $P$, et al. Metastasis in pulmonary arteries. J Radiol 2000; 81:807-809.

8. Travis WD, Brambilla E, Noguchi M, et al. Diagnosis of Lung Cancer in Small Biopsies and Cytology. Implications of the 2011 International Association for the Study of Lung Cancer/American Thoracic Society/European Respiratory Society Classification. Arch Pathol Lab Med 2013; 137:668684. 